\title{
A rare non-oncological pancreatic mass: eosinophilic pancreatitis diagnosis through EUS-FNA
}

\section{다)(웅}

\author{
Authors \\ Diogo Turiani Hourneaux De Moura ${ }^{1}$, Rodrigo Silva de Paula Rocha ${ }^{1}$, José Jukemura ${ }^{1}$, Vitor Ottoboni Brunaldi ${ }^{1}$, Hugo \\ Gonçalo Guedes ${ }^{1}$, Franz Robert Apodaca Torrez ${ }^{2}$, Igor Braga Ribeiro ${ }^{1}$, Andres Gelrud ${ }^{3}$, Eduardo Guimarães Hourneaux \\ De Moura ${ }^{1}$
}

Institutions

1 Hospital das Clínicas da Faculdade de Medicina da Universidade de São Paulo, Endoscopic Unit, Gastrointestinal Department, São Paulo, Brazil

2 Hospital São Paulo da Universidade Federal de São Paulo, Gastrointestinal Surgery Department, São Paulo, Brazil

3 Gastro Health and Baptist Health South Florida's Miami Cancer Institute, Florida, United States

submitted 14.6.2018

accepted after revision 2.9.2018

Bibliography

DOI https://doi.org/10.1055/a-0806-7099 |

Endoscopy International Open 2019; 07: E151-E154

(c) Georg Thieme Verlag KG Stuttgart · New York

ISSN 2364-3722
Corresponding author

Igor Braga Ribeiro, Rua Pais Leme, 215 - Ed. Thera Faria

Lima, Torre Água - Ap 1206, 05424-159, São Paulo/SP,

Brazil

Fax: +55-11-30697-579

igorbraga1@gmail.com

\section{ABSTRACT}

Background and study aims Eosinophilic pancreatitis $(E P)$ is a rare etiology of chronic pancreatitis, and few cases have been reported. It is characterized by eosinophilic infiltration of the pancreas and elevated IgE levels. EP is difficult to distinguish from pancreatic cancer based on clinical symptoms and auxiliary exams. We present a case of EP and debate the routine performance of endoscopic ultrasound-guided fine-needle aspiration (EUS-FNA) for resectable pancreatic mass.

\section{Introduction}

Eosinophilic pancreatitis (EP) is an extremely rare etiology of chronic pancreatitis, and only a few cases have been reported $[1,2]$. It is characterized by diffuse or localized eosinophilic infiltration of the pancreas and elevated IgE levels. Differential diagnosis between EP and pancreatic cancer (PC) is challenging because clinical symptoms and auxiliary exams (laboratory tests and radiological evaluation) may be rather similar [3].

Routine endoscopic ultrasound-guided fine-needle aspiration (EUS-FNA) for resectable pancreatic lesions is controversial [4]. Some experts advocate that routine FNA may detect a nonsurgical condition and therefore avoid an unnecessary operation and related complications. Conversely, EUS-FNA presents a substantial false-negative rate and may be associated with some adverse events, which might postpone or impair a curable resection $[5,6]$. We report a case that illustrates the aforementioned debate.

\section{Case report}

A 37-year-old man with a 4-month history of weight loss, obstructive jaundice, pruritus, and nausea and vomiting was admitted with an acute clinical complaint of fever, cough and dyspnea. He reported alcohol abuse for 15 years but denied any family medical history of PC. Physical examination revealed normal general appearance (body mass index $=21 \mathrm{~kg} / \mathrm{m}^{2}$ ) but jaundice $(2+/ 4+)$. Abdominal examination showed neither abdominal pain nor palpable masses. Laboratory tests were as follows: hemoglobin, $12.1 \mathrm{~g} / \mathrm{L}$; leukocyte count, $9.3 \times 10^{9} / \mathrm{L}$; neutrophils, $5.6 \times 10^{9}(56 \%)$; eosinophils: $0.12 \times 10^{9}(12 \%)$ (reference range: $0.0-0.06 \times 10^{9}$ ); total bilirubin $3.97 \mathrm{mg} / \mathrm{dL}$ (direct bilirubin: $3.52 \mathrm{mg} / \mathrm{dL}$ ); alkaline phosphatase, $769 \mathrm{U} / \mathrm{L}$; gammaglutamyl transferase, $591 \mathrm{U} / \mathrm{L}$; AST, $76 \mathrm{U} / \mathrm{L}$; ALT, $72 \mathrm{U} / \mathrm{L}$; and normal CA, 19.9. Hepatitis serology and autoimmune tests were negative.

Abdominal ultrasound showed biliary dilation but no cause for obstruction. Magnetic resonance cholangiopancreatography revealead biliary dilation due to an abrupt irregular narrowing in the distal common bile duct (CBD) without evident lesion 


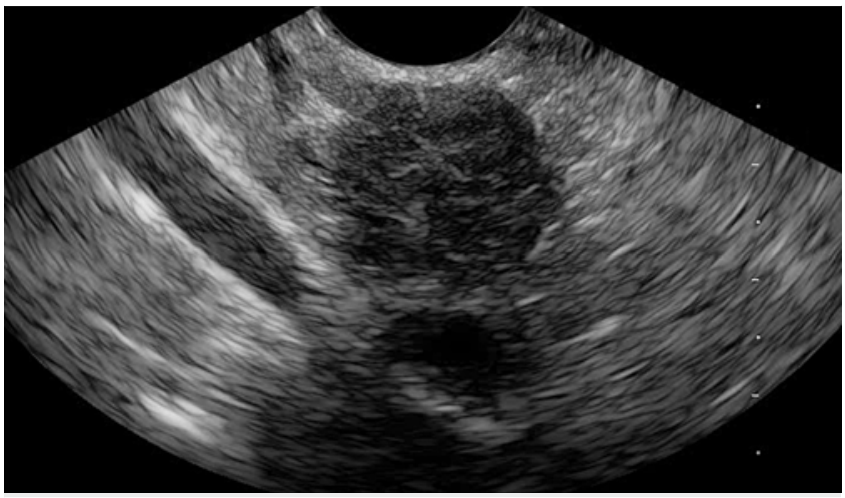

- Fig. 1 EUS image showing a hypoechoic pancreatic head mass.

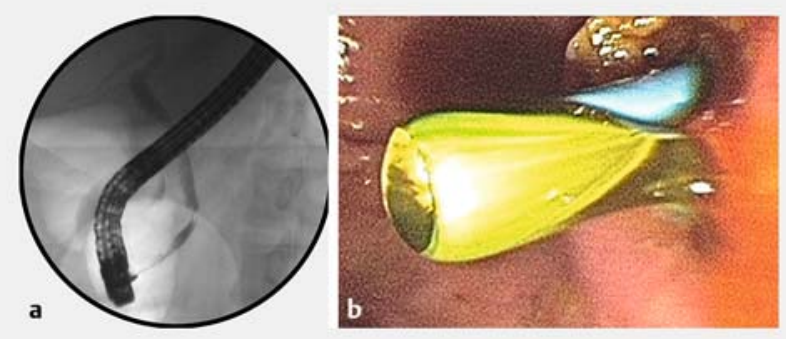

- Fig. 2 a ERCP showing a distal biliary stricture; b Biliary drainage with $10 \mathrm{Fr} \times 7 \mathrm{~cm}$ plastic stent.

associated with pancreatic body and tail atrophy and a normal pancreatic duct. Finally, abdominal computed tomography (CT) showed a $20-\mathrm{mm}$ mass in the head of the pancreas, hypoattenuating, with no vascular invasion ( $\bullet$ Fig. 1 ).

Endoscopic ultrasound (EUS) followed by endoscopic retrograde cholangiopancreatography (ERCP) to evaluate the mass and perform biliary drainage was indicated. EUS demonstrated a hypoechoic pancreatic head mass measuring $20 \mathrm{~mm}$ and a CBD dilatation. EUS-FNA was performed with a 22-gauge needle (Expect Slimline, Boston Scientific, Marlborough, Massachusetts, United States) but cytology was inconclusive.

ERCP demonstrated mild biliary duct dilatation due to a regular distal biliary stricture, suggesting pancreatic extrinsic compression ( $\triangleright$ Fig. 2a). Transpapillary forceps biopsy and brush cytology were negative for malignancy. A $10 \mathrm{Fr} \times 7-\mathrm{cm}$ plastic stent placement ensured adequate CBD drainage ( $\bullet$ Fig. $\mathbf{2 b}$ ).

After the negative sample results, a multidisciplinary group discussed surgery versus a new attempt at EUS-FNA. An EUSFNA was indicated, and once again, we used a 22-gauge needle (Expect Slimline, Boston Scientific, Marlborough, Massachusetts, United States). The new cytological evaluation showed massive pancreatic eosinophilic infiltration without a considerable increase in the presence of $\mathrm{IgG} 4$ positive cells, consistent with eosinophilic pancreatitis ( $\bullet$ Fig. 3 ).

After the diagnosis, the patient was also diagnosed with eosinophilic pneumonia and treated with systemic corticosteroids for 5 weeks.

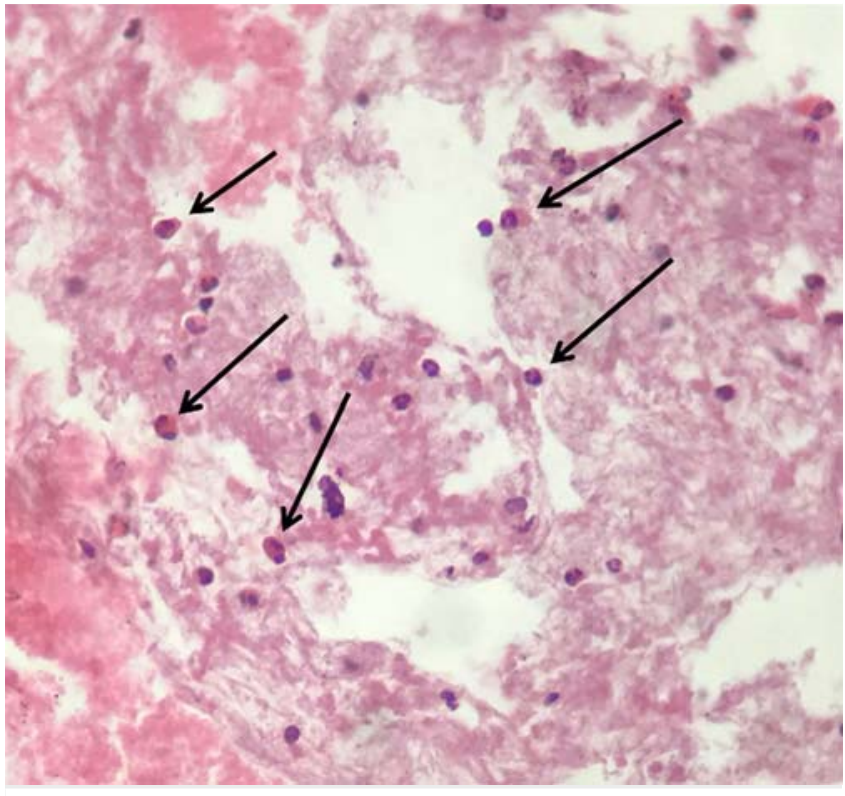

- Fig. 3 Cell block, hematoxylin-eosin staining, $400 \times$ Zoom, showing eosinophils in the pancreas.

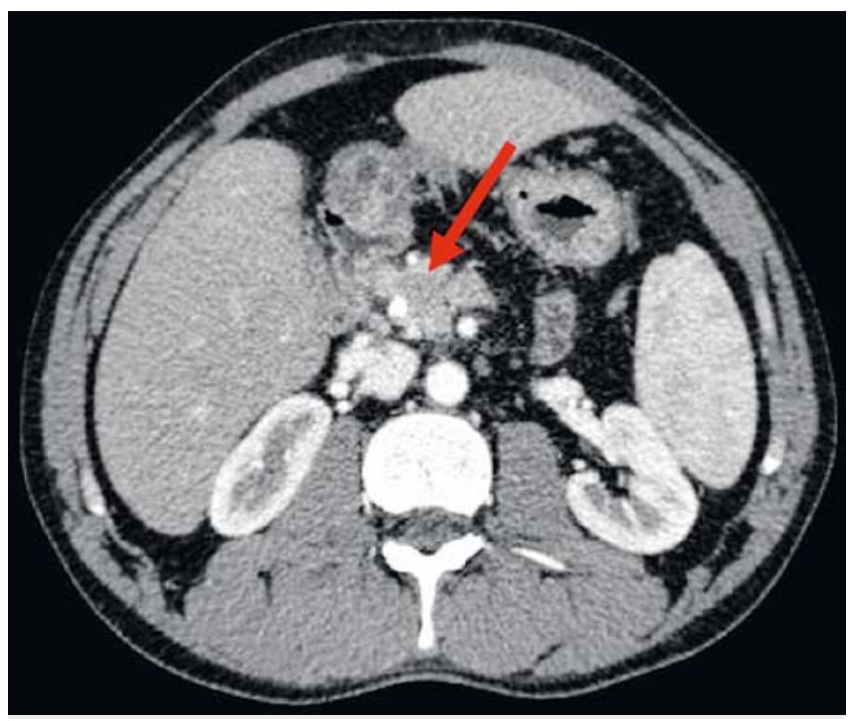

- Fig. 4 Post-treatment CT study revealing a mass shrinking and no biliary dilation.

Post-treatment contrast abdominal $\mathrm{CT}$ revealed mass shrinking from $20 \mathrm{~mm}$ to $12 \mathrm{~mm}$ without biliary tract dilation ( $\vee$ Fig. 4). Repeat complete blood count showed no elevation of serum $\operatorname{lgG} 4(121 \mathrm{mg} / \mathrm{dL})$, hemoglobin $16.0 \mathrm{~g} / \mathrm{L}$, leukocyte count $8.3 \times 10^{9} / \mathrm{L}$, neutrophils $4.6 \times 10^{9}(46 \%)$, eosinophils: $0.02 \times 10^{9}(2.0 \%)$, total bilirubin $0.37 \mathrm{mg} / \mathrm{dL}$, alkaline phosphatase $63 \mathrm{U} / \mathrm{L}$, and gamma-glutamyl transferase $132 \mathrm{U} / \mathrm{L}$.

At 1-year follow-up, the patient is asymptomatic on a low dose of systematic corticosteroids even after biliary stent removal. 
- Table 1 Distinct characteristics of eosinophilic and autoimmune pancreatitis.

\begin{tabular}{|l|l|l|}
\hline & Autoimmune pancreatitis & Eosinophilic pancreatitis \\
\hline Aspect of the pancreas & Enlarged pancreas (“sausage-like”) & Focal or diffuse \\
\hline Histopathological findings & Lymphocytes & Eosinophils \\
\hline Immunoglobulins & Elevated serum IgG4 & Elevated serum IgE \\
\hline Autoimmune and antinuclear antibodies & $(+)$ & $(-)$ \\
\hline
\end{tabular}

\section{Discussion}

EP is a rare etiology of chronic pancreatitis characterized by localized or diffuse eosinophilic infiltration of the pancreas and increased serum IgE [3]. Usually, the patient presents with a pancreatic mass due to eosinophilic inflammatory infiltration associated with obstructive jaundice, as in our case [2,3]. Many etiologies for EP have been suggested and published case reports include malignancy, parasitic infection, hypersensitivity to medications (e.g., carbamazepine), milk allergy, atopic diseases, newborn infant of a diabetic mother, and as an association with hypereosinophilic syndrome or eosinophilic gastroenteritis [1,7].

A diagnosis of EP is often made based on hypereosinophilic syndrome or eosinophilic gastroenteritis criteria in patients presenting with gastrointestinal symptoms who exhibit eosinophilic infiltration in histological/cytological samples $[3,4,8]$.

Diagnostic criteria for hypereosinophilic syndrome are as follows: peripheral eosinophil count higher than $1.5 \times 10^{9}$ for 6 months; past medical history of rhinitis, asthma or other allergic diseases; eosinophilic infiltration of other organs (digestive system, skin and/or heart); nad exclusion of other causes for eosinophilia such as parasitic infestations or leukemia $[3,8]$.

Regarding the eosinophilic gastroenteritis, which our patient had as well, the diagnostic criteria include presence of abdominal pain, vomiting, nausea, diarrhea, loss of appetite or any other digestive symptom; intestinal sampling showing eosinophilic infiltration and ruling out parasitic infection; and no involvement of organs outside the gastrointestinal tract [4].

The main differential diagnosis includes autoimmune pancreatitis (AIP) and PC [3].

EP is difficult to distinguish from AIP because they present with similar clinical symptoms and radiologic results $[3,7]$. However, subtle differences may favor one over the other. $>$ Table 1 outlines characteristics that may help differentiate EP from AIP.

Both EP and AIP are commonly misdiagnosed as PC due to the similarity of symptoms and imaging findings. It is important to recognize that some cases of PC are associated with eosinophilia, which can make the diagnosis of either condition difficult [3].

Definitive diagnosis depends on histological/cytological findings, assessment of serum tumor marker, absence of pancreatic duct dilation, history of hypereosinophilic syndrome or eosinophilic gastroenteritis, and response to corticosteroid or/ and cromolyn therapy $[4,9]$.
Nearly $7 \%$ of patients undergoing pancreatoduodenectomy for suspected malignancy are ultimately diagnosed with benign disease. Although some preoperative findings such as abdominal pain, absence of jaundice, normal pancreatic duct and absence of a mass or double duct sign on CT might indicate a benign condition, they are not sufficient to warrant use of conservative therapy [9]. EUS-FNA is an essential procedure for a patient with suspected PC who does not fit the typical demographics/preoperative findings.

Most cases of EP are diagnosed postoperatively. To avoid such unnecessary surgeries, adequate assessment of the patient's medical history and EUS-FNA for tissue sample are fundamental [3,6]. In our case, even after a negative EUS-FNA, we decided on another FNA because the patient was young and well-appearing, and had no dilation of the main pancreatic duct. However, only after the cytological results were we able to correlate the findings of the patient's clinical history and laboratory tests and diagnosed the hypereosinophilic syndrome.

There are few reports in the literature of cases of eosinophilic pancreatitis diagnosed by EUS-FNA $[1,8,10]$. As in our patient, adequate control of the disease was achieved using prednisolone or cromolyn $[3,10]$.

\section{Conclusions}

In summary, EP is a rare condition that is frequently misdiagnosed with PC. Clinical history, laboratory tests, radiological exams and tissue sampling are necessary for a definite diagnosis. Correct and precise diagnosis is crucial and may prevent unnecessary surgery and allow for noninvasive treatment. EUS-FNA is fundamental if the patient does not fit the typical findings for PC.

\section{Competing interests}

None

\section{References}

[1] Kakodkar S, Omar H, Cabrera J et al. Eosinophilic pancreatitis diagnosed with endoscopic ultrasound. ACG Case Rep J 2015; 2: 239-241

[2] Barthet M, Hastier P, Buckley MJ et al. Eosinophilic pancreatitis mimicking pancreatic neoplasia: EUS and ERCP findings-is nonsurgical diagnosis possible? Pancreas 1998; 17: 419-422 
[3] Tian L, Fu P, Dong X et al. Eosinophilic pancreatitis: Three case reports and literature review. Mol Clin Oncol 2016; 4: 559- 562

[4] Le Connie D, Nguyen H. Eosinophilic gastroenteritis, ascites, and pancreatitis: a case report and review of the literature. South Med J 2004; 97: 905-906

[5] Brugge WR. Fine needle aspiration of pancreatic masses: the clinical impact. Am J Gastroenterol 2002; 97: 2701-2702

[6] De Moura DT, Chacon DA, Tanigawa R et al. Pancreatic metastases from ocular malignant melanoma: the use of endoscopic ultrasoundguided fine-needle aspiration to establish a definitive cytologic diagnosis: a case report. J Med Case Rep 2016; 10: 332
[7] Barresi G, Inferrera C, De Luca F. Eosinophilic pancreatitis in the newborn infant of a diabetic mother. Virchows Arch A Pathol Anat Histol 1978; 380: $341-348$

[8] Valent P, Klion AD, Horny HP et al. Contemporary consensus proposal on criteria and classification of eosinophilic disorders and related syndromes. J Allergy Clin Immunol 2012; 130: 607-612.e9

[9] Gerritsen A, Molenaar IQ, Bollen TL et al. Dutch Pancreatic Cancer Group. Preoperative characteristics of patients with presumed pancreatic cancer but ultimately benign disease: a multicenter series of 344 pancreatoduodenectomies. Ann Surg Oncol 2014; 21: 3999 4006

[10] Song JW, Kim MH, Seo W] et al. [A case of eosinophilic pancreatitis]. Korean J Gastroenterol 2003; 42: 444-450 